\title{
Hamster Fibrosarcoma
}

National Cancer Institute

\section{Source}

National Cancer Institute. Hamster Fibrosarcoma. NCI Thesaurus. Code C134992.

Fibrosarcoma that occurs in a hamster. 